\title{
Modelling foam improved oil recovery within a heterogeneous reservoir
}

\author{
Elizabeth Mas Hernández ${ }^{\mathrm{a}}$, Paul Grassia ${ }^{\mathrm{b}, \mathrm{c}, *}$, Nima Shokri ${ }^{\mathrm{a}}$ \\ ${ }^{a}$ CEAS, The Mill, University of Manchester, Oxford Rd, Manchester M13 9PL, UK \\ ${ }^{b}$ Dept of Chemical and Process Engineering, University of Strathclyde, \\ James Weir Building, 75 Montrose St G1 1 XJ, UK \\ ${ }^{c}$ Departamento Ciencias Matemáticas y Fúsicas, Universidad Católica de Temuco, \\ Rudecindo Ortega 02950, Temuco, Chile
}

\begin{abstract}
The displacement of foam within a heterogeneous reservoir during foam improved oil recovery is described with the pressure-driven growth model. The pressure-driven growth model has previously been used to study foam motion for homogeneous cases. Here the foam model is modified in such a way that it includes terms for variable permeability. This model gives the evolution of the foam motion over time and the shape of the foam front, a wet foam zone between liquid-filled and gas-filled zones. The foam front shape for a heterogeneous or stratified reservoir develops concave and convex regions. For shapes such as these, the numerical solution of pressuredriven growth requires special numerical techniques, particularly in the case where concavities arise. We also present some analysis of the level of heterogeneity and how it affects the displacement, the shape of the front developing a set of concave corners. In addition to this we consider a heterogeneous and isotropic reservoir, in which case the foam front can sustain concavities, without these concavities having the same tendency to develop into corners.
\end{abstract}

Keywords: Improved Oil Recovery; Foam in Porous Media; Pressure-driven Growth; Heterogeneity; Anisotropy; Mathematical Modelling;

\section{Highlights}

* Pressure-driven growth model for foam improved oil recovery is considered

\footnotetext{
${ }^{*}$ Corresponding author
} 
* Reservoir is heterogeneous (stratified) and possibly also anisotropic

* Heterogeneity produces convexities and concavities in the foam front

* Concavities focus into corners that propagate differently from the rest of the front

* Exceedingly anisotropic systems give sharply-curved concavities but not corners

\section{Introduction}

Only a fraction of oil in a petroleum reservoir can be extracted under the reservoir's own pressure. To maintain the flow of oil, fluids must be injected into the reservoir to maintain the pressure. Owing to their special rheological properties foams are able to improve sweep efficiency during oil production processes from underground formations compared to other injection fluids [1]. Foams achieve this higher efficiency sweep efficiency by reducing gas mobility which, prevents injected gas from simply rising to the top of the reservoir where it would fail to displace the oil; and also suppresses viscous fingering, whereby injected fluid would simply follow established flow paths. However, many studies have focused on homogeneous formations even when in real fields heterogeneous conditions are found $[2,3]$. A heterogeneous formation has variable permeability [4]. Therefore, the flow of fluids, and in this specific case, of foams is affected by this difference in permeability.

There are some laboratory and simulation studies on heterogeneous reservoirs which give insight into the advantage of using foam as a displacing fluid for oil recovery $[2,5-10]$. They have found that foam is able to divert the gas flow from high permeability regions towards zones with lower permeability [2, 8-10].

Here we present a simulation study about the flow of foams within a heterogeneous reservoir. We have studied previously the displacement of foam within a homogeneous reservoir [11] (revisiting a model of Shan and Rossen [12]), and also two additional cases, one where the injection pressure is increased part way through the process and one taking into account the effect of surfactant slumping due to gravity $[13,14]$.

The mathematical description of the system for the homogeneous case uses a foam model known as pressure-driven growth $[11,12]$. The model computes the 
advance of a foam front which forms the boundary between liquid ahead and foam behind. Motion of the foam front is driven by pressure difference across the front, i.e. the difference between a driving injection pressure and the hydrostatic pressure in the liquid. The front speed falls as depth increases because the hydrostatic pressure rises. The front speed also falls the further the foam displaces: this is because most of the dissipation in the system occurs in a wet foam region where the foam meets the liquid, and this wet foam region thickens over time, but its thickness always remains much less than the distance over which the front itself propagates $[11,12,15]$. The two additional cases, mentioned above i.e. increase in driving pressure and surfactant slumping, use a suitable modification of the same model $[13,14]$. Therefore, in a similar fashion, we propose some changes to the original pressure-driven growth model that will make it appropriate to describe the case of the heterogeneous reservoir.

The rest of this work is structured as follows: section 2 describes the changes we have applied to the pressure-driven growth model to make it suitable for a heterogeneous reservoir. Section 3 presents results of the numerical solution of the system highlighting some numerical implementation issues. Quantitative comparison between homogeneous and heterogeneous fronts is addressed in sections 4 and 5 , which give insight into the behaviour of the heterogeneous displacement. We have also explored the case of a heterogeneous but anisotropic reservoir, this is shown in section 6. Finally, section 7 offers conclusions.

\section{Pressure-driven growth for variable permeability}

The sketch in Figure 1 illustrates the system that is considered via the pressuredriven growth model here. The physical content of the pressure-driven growth model (regardless of whether in a homogeneous or heterogeneous case) is that the speed of a foam front is proportional to the net driving pressure difference across it, this being the difference between the injection pressure and the hydrostatic pressure in the reservoir. Since hydrostatic pressure grows linearly with depth, the net driving pressure decays as a straight line function with depth, falling to zero at a critical depth. 
For the description of the heterogeneous case, the pressure-driven growth model ${ }^{1}$ has been modified in a simple way: basically this takes into account permeability variation.

We describe relative changes in permeability with the help of a sinusoidally varying function, given below, which represents the reservoir heterogeneity and is included within the equations describing the speed of the foam displacement.

Therefore the equations that apply in this case for horizontal and vertical components of velocity $\operatorname{are}^{2}$ :

$$
\begin{aligned}
& \frac{\mathrm{d} X_{D}}{\mathrm{~d} t_{D}}=\frac{\left(1-Z_{D}\right) \cos \alpha}{s_{D}} J\left(Z_{D}\right) \\
& \frac{\mathrm{d} Z_{D}}{\mathrm{~d} t_{D}}=\frac{\left(1-Z_{D}\right) \sin \alpha}{s_{D}} J\left(Z_{D}\right)
\end{aligned}
$$

where to illustrate the model $J\left(Z_{D}\right)$ can be chosen to be one of the following functions:

$$
\begin{aligned}
& J\left(Z_{D}\right)=1+k_{s} \sin \left(2 \pi n_{s} Z_{D}\right) \\
& J\left(Z_{D}\right)=1-k_{s} \sin \left(2 \pi n_{s} Z_{D}\right)
\end{aligned}
$$

$X_{D}$ is the horizontal position of a material point in a rectangular reservoir, $Z_{D}$ the vertical position downwards, $1-Z_{D}$ represents the decay of net driving pressure with depth, $t_{D}$ is time, $s_{D}$ is the distance material points on the front travel, $\alpha$ the angle giving the orientation of the front normal with respect to the horizontal, $k_{s}$ is the amplitude of the heterogeneity variation about the mean (a factor less than unity), and $n_{s}$ is the number of low and high permeability layers (for simplicity taken to be an integer). The reason for choosing a sinusoidal variation for the spatial variation of the permeability is so that the wavelength of the sinusoid can match the length scale of the layers in a heterogeneous stratified reservoir.

The above equations need to be solved with suitable boundary and initial conditions. The boundary condition is that motion needs to be horizontal along the top of the reservoir, so that $\alpha=0$ at $Z_{D}=0$. The initial condition is that the front is

\footnotetext{
${ }^{1}$ Refer to $[11,12]$ for the mathematical description of the model for constant permeability.

${ }^{2}$ These equations are in dimensionless form. Dimensional equations for the original system and their conversion to the dimensionless version are given in the Appendix.
} 
initially vertical and located at $X_{D}=0$ for all $Z_{D}$. In addition $s_{D}$ is initially zero for all material points, but grows as those material points displace.

Contrary to the convex shape expected for displacement in a homogeneous reservoir, in the present case the foam front shape will develop alternate convex and concave regions. This is because when the sinusoidally-varying permeability function affects the velocity of the front motion, describing the propagation through the different layers with high and low permeability, velocity becomes respectively faster and slower than a system with unit permeability.

Owing to the nature of the pressure-driven growth model, the formation of concavities during the course of the evolution will lead to numerical difficulties, including predictions of spurious loops in the shape of the front [11].

In order to handle concave regions and avoid the formation of loops, velocity displacement is corrected as in [11]: concave regions are set to move with an apparent velocity $v_{a p p}=v / \cos (\theta / 2)(v$ is the front speed and $\theta$ is the angle through which the front turns between adjacent segments, see Figure 1), when the angle $\theta$ exceeds a certain threshold given by $\theta_{s}$. In line with previous work [11] we take $\theta_{s}$ to be a constant significantly smaller than unity specifically with the value $\pi / 18$. Setting $v_{\text {app }}=v / \cos (\theta / 2)$ will speed up the displacement of the points with the aim of catching up with points which have displaced further ahead. The reason why the apparent velocity $v_{a p p}$ takes the above mentioned form has been explained on physical and geometric grounds by [11]: concavities can focus down into sharp corners and these need to propagate at a higher speed than points nearby to prevent indefinite sharpening. Physically these sharp corners are idealisations of regions in which the orientation of the foam front changes significantly over a length scale comparable with the thickness of the wet foam front, which (as was stated previously) is much smaller than the length scale over which the front itself propagates.

In the above, we have shown the main changes applied to the mathematical model for pressure-driven growth. The next section presents numerical results without and with implementation of velocity corrections for concavities. Details of numerical implementation issues within the system are also given. 


\section{Numerical results for displacement with variable permeability}

When solving the system for pressure-driven growth taking into account variable permeabilities, as described by equations (1)-(2) with any given values for the parameters $k_{s}$ and $n_{s}$, the resulting front shape has smooth convex and concave regions at short times for either of the $J\left(Z_{D}\right)$ functions presented previously.

At later times, concavities focus down to sharp corners and (unless the velocity at each corner is corrected as alluded to above) these develop spurious loops [11]. Also, highly curved convex regions can be formed at the top of the domain [11]. These are associated with the boundary condition usually imposed at the top boundary, namely $\alpha=0$. Material points near the top of the domain with $\alpha$ close to zero tend to migrate downwards only very slowly, but as they migrate, they also fall increasingly behind the leading edge of the front on the top boundary itself, thereby producing high curvatures. This results in material points migrating even further down and leaving only a few points at the top region of the foam front thereby giving a poor representation of its shape.

We present numerical results, first for the original system (without special features to handle the formation of loops and sharp concavities) in section 3.1, and then implementing modifications to deal with these issues (section 3.2).

\subsection{Numerical results for original system}

Figure 2(a) shows some results when the front is discretised using 500 points along the $Z_{D}$ axis, the time step is $1 \times 10^{-5}, k_{s}=0.3$, and $n_{s}=3$ using equation (3). The values for $k_{s}$ and $n_{s}$ are chosen arbitrarily for the purpose of illustrating the model. Also, the segment at the top of the front is subdivided if its length reaches a value equal to 0.01 [11]. Note that an earlier study [11] on a homogeneous system (as opposed to a heterogeneous one) reported results using 40 spatial intervals, a time step $5 \times 10^{-5}$, with a threshold for subdividing the top interval of 0.05 . In the present study to ensure that the effects of heterogeneity can be resolved, the spatiotemporal resolution has been chosen roughly an order of magnitude better than that previous study. In Figure 2(a) a spurious loop is clearly evident for $t_{D}=0.2$ and even for $t_{D}=0.14$ a tiny loop can be seen. Similarly, Figure 2(b) shows results for the same 
parameters but using equation (4). In this case the loop is formed earlier: already by time $t_{D}=0.1$ a very significant loop is in evidence ${ }^{3}$.

We can see from these figures that, due to the nature of the model, the effect of high and low permeability layers on the foam front shape seems more evident closer to the top of the front.

Near the bottom (of the domain of solution), the concavity formed is weak and points in this region migrate downwards where the displacement of the front is null: as the front reorients over time, owing to the net driving pressure (i.e. injection pressure less hydrostatic pressure) decreasing with depth, material points tend to move downwards as well as to the right, so that any weak concavities that develop near the bottom of the front then migrate further and further down towards a region where the velocity is virtually nil.

Actually, this behaviour of the front displacement is what makes the concavity formed closer to the top of the domain, due to a local minimum permeability value, to evolve faster into a spurious loop than the next low permeability layer further down.

Figure 2(a) shows a concavity forming around about $Z_{D}=0.3$, but above this (i.e. at smaller $Z_{D}$ values) there is a convexity around about $Z_{D}=0.1$. This 'local' convexity has higher curvature than the corresponding convex front shape that arises in a system with homogeneous permeability [11]. As a result, material points in this convexity separate comparatively quickly from one another, and hence over time (and without adding extra material points) the foam front starts to look quite jagged.

Moreover it appears that the jagged foam front in Figure 2(a) fails to satisfy the boundary condition imposed at the top boundary (the front is supposed to meet that boundary at right angles). We can make the foam front less jagged by subdividing the intervals between material points, particularly those intervals in the

\footnotetext{
${ }^{3}$ In the case of a homogeneous reservoir presented by [11], and deliberately inserting a concavity into the otherwise convex shape, it takes an order of magnitude longer to develop into a problematic loop.
} 
neighbourhood of this convexity near $Z_{D}=0.1$ That means in turn that intervals on these local convexities need to be subdivided, just as in the homogeneous reservoir case we needed to subdivide the topmost interval ${ }^{4}$. Therefore, strategies to regrid the foam front are also needed to have a better representation of its shape. In concave regions, material points actually move closer together instead of separating, and this means that we have to remove material points when regridding instead of adding new ones.

The following section presents results when the implementation of speed up for concavities and front regridding are used.

\subsection{Modifying velocity for concavities and regridding the front}

To avoid the concavities focussing down to sharp corners and forming spurious loops, velocities must be modified for concave regions (using a speed up factor $1 / \cos (\theta / 2)$ as explained in section 2: this speed up effect is what causes the sharpness of the corners to saturate). Also we have used a linear interpolation for regridding the front as a first approximation.

Figures 3(a)-3(b) present foam front displacement, respectively for the two $J\left(Z_{D}\right)$ functions, now modifying velocities for concave regions and implementing the front regridding. For the plots in these figures, 500 material points are used to discretise the front, time step is $1 \times 10^{-5}, k_{s}=0.3, n_{s}=3$, the parameter $\theta_{s}=\pi / 18$ is used as the critical angle for switching on the velocity modification (this value of $\theta_{s}$ is chosen arbitrarily but results are not sensitive to $\theta_{s}$, as long as it is much smaller than $\pi$ as explained by [11]), points between short spatial intervals (shorter than 0.002 ) are set to be consumed when implementing velocity corrections [11], regridding takes place when the front segments are longer than a specified value (0.02), and the topmost segment is subdivided when it becomes longer than 0.01.

The effect is to contain concavities, keeping them from developing into loops. In addition, having more points to describe the foam front ensures a less jagged

\footnotetext{
${ }^{4}$ There is a special rule that is used to subdivide the topmost front segment which arises due to the very high curvatures that can occur on the top boundary: see [11] for details.
} 
shaped front, away from the concave corner. We are also able to obtain results for longer time values. Figure 4 shows results at comparatively long times when the heterogeneity function is given by equation (4).

It is interesting to consider whether the profile might eventually attain an asymptotic state in the long time limit. Previous studies in the case of homogeneous permeability $[11,13,14]$ have demonstrated that the system attains a state in which the 'apparent' horizontal velocity is uniform with position. 'Apparent' horizontal velocity is the difference in horizontal displacement per difference in time between two points on the front at the same vertical height at two different times. It is not the same as the velocity of material points, because material points do not remain at a fixed vertical height. On the basis of equations (1)-(2) the apparent horizontal velocity [11] can be deduced to be $\left(1-Z_{D}\right) J\left(Z_{D}\right) /\left(s_{D} \cos \alpha\right)$ where geometry implies $\cos \alpha \equiv-\mathrm{d} Z_{D} / \mathrm{d} X_{D}\left(1+\left(\mathrm{d} Z_{D} / \mathrm{d} X_{D}\right)^{2}\right)^{-1 / 2}$. In the long time limit we can, with negligible error, we can replace all $s_{D}$ values by the corresponding value for the leading edge at the top of the front, $Z_{D}=0$, with in addition $J=1$ at this point which follows from (4). Imposing uniformity of the apparent horizontal velocity across all $Z_{D}$ leads to

$$
\frac{\mathrm{d} Z_{D}}{\mathrm{~d} X_{D}}=-\frac{\left(1-Z_{D}\right) J\left(Z_{D}\right)}{\sqrt{1-\left(\left(1-Z_{D}\right) J\left(Z_{D}\right)\right)^{2}}}
$$

and hence

$$
\frac{\mathrm{d} X_{D}}{\mathrm{~d} Z_{D}}=-\frac{\sqrt{1-\left(\left(1-Z_{D}\right) J\left(Z_{D}\right)\right)^{2}}}{\left(1-Z_{D}\right) J\left(Z_{D}\right)}
$$

from which the long-time asymptotic form of $X_{D}$ vs $Z_{D}$ can be obtained by quadrature. The sinusoidal oscillations present in the function $J\left(Z_{D}\right)$ will manifest themselves in the form of $X_{D}$ vs $Z_{D}$.

Returning to the numerical system, it is not readily possible within a numerical scheme to reach longer times when describing permeability variation with equation (3), at least not when using only the modifications described above. Points below the top boundary displace further in the horizontal direction than the topmost point when using this equation, in consequence the segments in this region develop negative curvature and points start migrating above $z_{D}=0$ and developing loops in this region. In such cases, we can impose a condition in the algorithm to discard 
points with vertical position $z_{D}<0$. This restriction solves this problem of course. Furthermore, in such a case we cannot set the orientation of the front along the horizontal top boundary (i.e. we can no longer satisfy the boundary condition $\alpha=0$ there). It is therefore necessary to calculate the horizontal location of the intersection of the front with $z_{D}=0$, instead of specifying the trajectory of the front at $z_{D}=0$ in advance as was done for an analogous homogeneous system in [11]. However we have not explored this situation in detail in the present study, leaving it instead for future study with more sophisticated numerical techniques [16].

We are interested in how results for the heterogeneous reservoir compare to the homogeneous one and in the effect of using different values for $k_{s}$ and $n_{s}$. To be able to measure this quantitatively, we perform calculations for the root mean square displacement between homogeneous and heterogeneous fronts, which is considered in the following section.

\section{Root mean square displacement between homogeneous and heteroge- neous fronts}

Root mean square displacement is obtained as follows. For each point in the homogeneous front the displacement (normal to this front) ahead of and behind it are followed until the intersection with the heterogeneous front is obtained. These displacements are squared and multiplied by their corresponding length of arc in the homogeneous curve, integrated, divided by total length of the curve for the homogeneous front and the square root of this amount is taken.

It is important to note that the number of material points is different in each front, heterogeneous versus homogeneous (the number of points for the heterogeneous front tends to decrease early on as points are consumed more rapidly than they are created; on the other hand the number of points for the homogeneous front does not quite so vary much). Point positions are also different on each front, in such a way that to calculate the displacement normal to the homogeneous curve it is necessary to interpolate (linearly) between points in the heterogeneous front for any given point on the homogeneous front because there is no guarantee that the 
normals to the discrete material points at the homogeneous curve will intersect the discrete points representing the heterogeneous curve.

Figures 5(a)-5(c) compare heterogeneous fronts to homogeneous ones for the function $J\left(Z_{D}\right)$ described by equation (4) for various $k_{s}$ and $n_{s}$. These plots are obtained discretising the homogeneous front with 100 points and the heterogeneous one with 200 points, the time step and criteria to correct velocities, and for remeshing, are the same as before.

Table 1 gives values for root mean square displacement between homogeneous and heterogeneous fronts at different time values and varying parameter $k_{s}$.

Figure 6(a) shows a plot of root mean square displacement against time for the values in Table 1 . In addition, we have also plotted the value for $t_{D}=0$ when both fronts coincide. Figure 6(b) presents the data for the displacement versus $k_{s}$.

These results allow us to confirm assumptions about how the displacement between homogeneous and heterogeneous fronts changes with different values for amplitude of heterogeneity. Root mean square displacement increases with increase in $k_{s}$. The reason for this is that with the increase in amplitude of heterogeneity, the heterogeneous front tends to displace further away (ahead and behind) from the homogeneous one.

Regarding the parameter $n_{s}$ for the number of high and low permeability layers, Table 1 also gives values for the root mean square displacement varying time and $n_{s}$. We have also these data in Figure 7, showing root mean square displacement against time in Figure 7(a) and against $n_{s}$ in Figure 7(b). These results show that root mean square displacement does not depend upon $n_{s}$, at least for the parameters and time values presented so far.

When comparing fronts between homogeneous and heterogeneous results, we are also interested in identifying points on the heterogeneous curve which are local maxima and minima of the displacement relative to the homogeneous one (normal to the homogeneous front). These points presumably develop in regions where permeability is maximum or minimum, but potentially they could migrate as material points themselves migrate. This is covered in the next section. 


\section{Local maxima and minima on heterogeneous front}

Observing the front for heterogeneous displacement in the previous figures, and specially when comparing them to the homogeneous ones (e.g. Figure 5(a)), it seems that for $n_{s}=3$ there are three local maxima ahead and three local minima behind on each curve along the normal to the homogeneous fronts, and that these points tend to migrate downwards over time.

Trying to test this hypothesis, we have calculated where these points are located and the displacement (the distance between the heterogeneous point and the homogeneous front along the normal to the homogeneous front) to check how they change over time for the data presented previously in Figure 5 (a) (at times $t_{D}=0.1,0.2$ and 0.3 , with $\left.k_{s}=0.3, n_{s}=3\right)$. Figure 8 shows similar data: segments normal to the homogeneous front for points which are local minima and maxima are also shown; and it is evident that there are 3 local minima and 3 local maxima alternately.

Figures $9(\mathrm{a})$ and $9(\mathrm{~b})$ present graphs of vertical locations $\left(Z_{D}\right)$ of the maximum and minimum points and the displacement separating those points from the homogeneous curve versus time, respectively. At these time values, for all local minimum and maximum points $Z_{D}$ increases (in Figure $9(\mathrm{a})$ ) but the rate of increase is comparatively low.

The distances between the maximum or minimum points and the homogeneous curve as shown in Figure 9(b) exhibit interesting behaviour. Whereas the distances between the maxima and the homogeneous curves always grow with time, that between the minima and the homogeneous curves sometimes shows decreases. The mechanism for decreasing the distance in the case of the minima is that once a concavity develops into a sharp concave corner, the corner is sped up relative to neighbouring material points. This then points to an interesting feature of the computed front shapes, which cannot be detected by the root mean square displacement statistic considered earlier, namely there is a fore and aft asymmetry between the heterogeneous and homogeneous curve. In Figures 5(a)-5(c) a significantly higher fraction of the heterogeneous curve lies in front of the homogeneous curve than lies behind it. If, at each time shown in those figures, one were to integrate the area 
that has been swept out by the foam front, it is evident that the heterogeneous front sweeps out larger area than the homogeneous one, even though by construction, the distance that both front advance along the top boundary is the same as the permeability at the top boundary is unchanged.

We reiterate that the maximum and minimum points identified in Figure 8 at different times are not always the same material point. We have also explored in Figure 10 downward migration of selected material points at early time in a homogeneous system. Comparing Figure 10 and Figure 9(a) we see that the downward displacement of the material points (homogeneous system) is substantially faster than that of the maxima and minima (heterogeneous system). This underlines the fact that the maxima and minima are geometric features of the heterogeneous front, but do not themselves correspond to trajectories of material points.

The foam displacement for a homogeneous reservoir but with anisotropic permeability has been studied by de Velde et al. [15]. Further results for anisotropic systems have been obtained by Grassia et al. [17] who specifically determined the speed of the concave corners relative to neighbouring material points (an anisotropic generalisation of the $1 / \cos (\theta / 2)$ speed up factor that was discussed previously). In the next section we also explore this case.

\section{Anisotropic reservoir}

For an anisotropic reservoir we consider that vertical and horizontal permeability differ from each other. Hence the ratio between permeabilities (denoted $k_{v}$ ) is no longer unity $\left(k_{v} \neq 1\right)$.

Velocity components in a homogeneous but anisotropic reservoir are described with the following equations [15]:

$$
\begin{gathered}
\frac{\mathrm{d} X_{D}}{\mathrm{~d} t_{D}}=\frac{\left(1-Z_{D}\right)}{s_{D}} \frac{\cos \alpha}{\cos (\alpha-\beta)} \\
\frac{\mathrm{d} Z_{D}}{\mathrm{~d} t_{D}}=k_{v} \frac{\left(1-Z_{D}\right)}{s_{D}} \frac{\sin \alpha}{\cos (\alpha-\beta)}
\end{gathered}
$$

where $k_{v}$ is the ratio of vertical to horizontal permeability and $\beta$ is the angle giving the direction (measured with respect to the horizontal) in which the front is moving 
for this case, as illustrated in Figure 11. The reason for the $\cos (\alpha-\beta)$ term in the denominator is that the front thickness (which determines the amount of dissipation in the system [12]) when measured along the direction $\beta$ grows proportionally to the distance travelled $s_{D}$, whereas the pressure gradient across the front scales inversely with the lesser thickness measured along the direction $\alpha$ (i.e. along the front normal).

For the heterogeneous case, equivalent equations to (7)-(8) are used but including a function $J\left(Z_{D}\right)$ for heterogeneity [17], in the same way as for the isotropic case presented in section 2. Therefore, velocity components are:

$$
\begin{gathered}
\frac{\mathrm{d} X_{D}}{\mathrm{~d} t_{D}}=\frac{\left(1-Z_{D}\right)}{s_{D}} \frac{\cos \alpha}{\cos (\alpha-\beta)} J\left(Z_{D}\right) \\
\frac{\mathrm{d} Z_{D}}{\mathrm{~d} t_{D}}=k_{v} \frac{\left(1-Z_{D}\right)}{s_{D}} \frac{\sin \alpha}{\cos (\alpha-\beta)} J\left(Z_{D}\right) .
\end{gathered}
$$

Some preliminary numerical results for the foam front shape over time (with $k_{v}=0.1$ ) and some numerical analysis for the heterogeneous anisotropic reservoir are presented in [17]. However the focus there was mostly on checking that the algorithms employed were able to avoid the formation of spurious loops. A detailed parametric study (varying heterogeneity parameter $k_{s}$ and contrasting anisotropic systems with isotropic ones) is not included there. Therefore, in what follows, we have obtained results for various values of $k_{s}$ (keeping $n_{s}=3$ ) comparing data for $k_{v}=0.1$ to $k_{v}=1$ (actually the latter is equivalent to an isotropic system). Comparing fronts at $k_{v}=0.1$ and $k_{v}=1$, in Figure 12(a) for small $k_{s}$ and short time the fronts look similar. Figure 12(b) shows that increasing $k_{s}$ and at longer time, the fronts do not coincide any more, the anisotropic displacement (with $k_{v}=0.1$ ) maintains smooth concave and convex regions for longer and these lag behind corners developed using $k_{v}=1$.

In addition, an earlier study dealing with the homogeneous anisotropic reservoir also presents an analytical solution for the front shape when the vertical permeability is identially zero [15]. Here we extend this approach and apply it to the heterogeneous reservoir.

When vertical permeability is zero, the system of differential equations to de- 
scribe (heterogeneous anisotropic) foam displacement is simplified to:

$$
\begin{gathered}
\frac{\mathrm{d} X_{D}}{\mathrm{~d} t_{D}}=\frac{\left(1-Z_{D}\right)}{s_{D}} J\left(Z_{D}\right) \\
\frac{\mathrm{d} Z_{D}}{\mathrm{~d} t_{D}}=0 \\
\frac{\mathrm{d} s_{D}}{\mathrm{~d} t_{D}}=\frac{\mathrm{d} X_{D}}{\mathrm{~d} t_{D}}
\end{gathered}
$$

from which

$$
\frac{\mathrm{d} X_{D}}{\mathrm{~d} t_{D}}=\frac{\left(1-Z_{D}\right)}{X_{D}} J\left(Z_{D}\right)
$$

which can be solved analytically, giving ${ }^{5}$ :

$$
X_{D}=\sqrt{2\left(1-Z_{D}\right) J\left(Z_{D}\right) t_{D}}
$$

Using either equation (3) or (4) for $J\left(Z_{D}\right)$, defining parameters $k_{s}, n_{s}$, and setting the time and values for $Z_{D}$ it is possible to calculate $X_{D}$ with (15). One consequence of having zero vertical permeability is that material points no longer move vertically, and hence no longer move down from the top boundary even if the front meets it obliquely, which implies that it is no longer necessary to impose a condition $\alpha=0$ on the top boundary (contrast with the situation in [11]).

Figure 13 depicts analytical results for both homogeneous and heterogeneous fronts in the limiting case of zero vertical permeability using equation (3) changing $k_{s}$ and $n_{s}$ for the heterogeneous front. Heterogeneous data are obtained using equation (15) and homogeneous points with $X_{D}=\sqrt{2\left(1-Z_{D}\right) t_{D}}$ (the analytical solution obtained by [15]).

We have also calculated root mean square displacements for the foam fronts presented in Figure 13, measuring displacement along the normal for points on the homogeneous front (as explained previously) but also measuring it along the horizontal (which is easy to determine since all motion is horizontal in the case when vertical permeability is identically zero). Results (although not reproduced

\footnotetext{
${ }^{5}$ Note that since $J\left(Z_{D}\right)$ is a smooth function here, equation (15) necessarily describes a smooth curve without any sharp corners. For sharp corners to develop, vertical permeability must be non-zero: material points must move both horizontally and vertically.
} 
here) show that root mean square displacement, either along the normal or the horizontal, increases with $k_{s}$. Changes in the root mean square displacement with respect to $n_{s}$ turn out to be very small; so we can conclude that this parameter does not influence root mean square displacement.

We have also compared numerical results for $k_{v}=0.1$ to the analytic solution with zero vertical permeability for parameters $k_{s}=0.1,0.3,0.4,0.5$. Analytic results obtained with equation (15) seem to match closely with $k_{v}=0.1$ numerics for any of the explored values of $k_{s}$ and $t_{D}$. This is consistent with the findings of [15] in the limit of homogeneous and isotropic systems. Figure 14 shows results for $t_{D}=0.3$ and $k_{s}=0.5$, which is analogous to what was considered in Figure 12(b).

\section{Conclusions}

We have shown that it is possible to use a simple adaptation of the pressuredriven growth model for a heterogeneous stratified reservoir. A sinusoidal function is used to describe permeability variation. The shape of the foam front in this case develops convex and concave regions making it quite distinct from the shape predicted in a homogeneous reservoir. The computational algorithm prevents concavities from forming into spurious loops, and concavities are contained using a factor to correct speed of points in concave sections. At longer times we still obtain a good representation of the front shape for which convex regions are joined together at corners, which themselves become less sharp towards the bottom of the front. It is possible to compare quantitatively results for the heterogeneous case to data for the homogeneous reservoir. For this we calculated root mean square displacement, which increases with the amplitude of heterogeneity $\left(k_{s}\right)$ and with time, and is not sensitive to the wavenumber $\left(n_{s}\right)$. Points at the heterogeneous front which are local maxima and minima with respect to the distance from the homogeneous front move downwards, at least for the small values of time $t_{D}$ considered here. The rate of downward migration of these maxima and minima is however much smaller than that of material points, and indeed we would expect to see downward drift of these maxima and minima saturate for a long-time asymptotic front shape. A fore-to-aft 
asymmetry is also observed in the heterogeneous fronts, with more points in the heterogeneous case running ahead of the homogeneous front than lagging behind it. This comes about owing to concave corners at the minima moving faster than material points do, reducing the amount that the corners would otherwise lag behind. The implication is that the heterogeneous foam front sweeps out more area than the homogeneous front does. We have also obtained an analytical solution for a heterogeneous but anisotropic reservoir for the limit case of zero vertical permeability and compared these data to some numerical results and to a homogeneous anisotropic formula. Results show agreement between numerical and analytical front shapes, particularly when the level of anisotropy is high (i.e. when the ratio $k_{v}$ of vertical to horizontal permeability is small).

\section{Appendix}

The foam front displacement for a homogeneous reservoir has been described with a simplified model (pressure-driven growth) proposed by Shan and Rossen [12] that keeps track of material points $(x(t), z(t))$, where $x$ and $z$ are horizontal and vertical point positions, $t$ is time; and $s(t)$ is the distance that individual points travel. Dimensional equations for horizontal and vertical velocity components are:

$$
\begin{aligned}
\frac{\mathrm{d} x}{\mathrm{~d} t} & =\frac{k \lambda_{r f}|\nabla P| \cos \alpha}{\left(1-S_{w f}\right) \phi} \\
\frac{\mathrm{d} z}{\mathrm{~d} t} & =\frac{k \lambda_{r f}|\nabla P| \sin \alpha}{\left(1-S_{w f}\right) \phi}
\end{aligned}
$$

with

$$
\begin{aligned}
& |\nabla P|=\frac{\Delta P-\Delta \rho g z}{\tau} \\
& \alpha=\arctan \left(-\frac{\partial x}{\partial z}\right)_{t}
\end{aligned}
$$

and

$$
\frac{\mathrm{d} s}{\mathrm{~d} t}=\sqrt{\left(\frac{\mathrm{d} x}{\mathrm{~d} t}\right)^{2}+\left(\frac{\mathrm{d} z}{\mathrm{~d} t}\right)^{2}}
$$

where $k$ is permeability, $\lambda_{r f}$ is gas relative mobility in the foam front region, $\nabla P$ is pressure gradient, $\Delta P$ is injection pressure less hydrostatic, $\alpha$ is front orientation, $S_{w f}$ is water saturation in the foam front region, $\phi$ is porosity, $\Delta \rho$ is liquid to gas 
density difference, $g$ is acceleration due to gravity, and $\tau$ is the width of the front, assumed to be proportional to the distance the front has displaced.

Distances $x, z$ and $s$ are made dimensionless dividing by $\Delta P / \Delta \rho g$. Time is made dimensionless using:

$$
t_{D}=\frac{k \lambda_{r f} \Delta \rho g}{\left(1-S_{w f}\right) \phi \tau^{*}} t
$$

where $\tau^{*}$ is a characteristic front width (when the front has displaced a distance equal to $\Delta P / \Delta \rho g)$.

When equations (16) and (17) are non-dimensionalised based on these scales, and subsequently generalised to the case of heterogeneous permeability, equations (1) and (2) result.

\section{Acknowledgements}

EMH acknowledges scholarship funding from the EPS-CONACyT programme. PG acknowledges funding from CONICYT Chile (folio 80140040).

\section{References}

[1] L. L. Schramm, J. J. Novosad, Micro-visualization of foam interactions with a crude oil, Colloids and Surfaces 46 (1) (1990) 21-43.

[2] H. J. Bertin, O. G. Apaydin, L. M. Castanier, A. R. Kovscek, Foam flow in heterogeneous porous media: Effect of cross flow, SPE Journal 4 (2) (1999) $75-82$.

[3] U. G. Araktingi, F. M. Orr Jr, Viscous fingering in heterogeneous porous media, SPE Advanced Technology Series 1 (1) (1993) 71-80.

[4] C. Zhang, M. Oostrom, J. W. Grate, T. W. Wietsma, M. G. Warner, Liquid $\mathrm{CO}_{2}$ displacement of water in a dual-permeability pore network micromodel, Environmental Science \& Technology 45 (17) (2011) 7581-7588.

[5] W. J. Renkema, W. R. Rossen, Success of SAG foam processes in heterogeneous reservoirs, in: SPE Annual Technical Conference and Exhibition, Nov 11-14, 2007, Anaheim, California, U.S.A., 2007. 
[6] Y. Zhang, X. Yue, J. Dong, L. Yu, New and effective foam flooding to recover oil in heterogeneous reservoir, in: 2000 SPE/DOE Improved Oil Recovery Symposium, April 3-5, 2000, Tulsa, Oklahoma, Society of Petroleum Engineers, 2000.

[7] M. B. Kloet, W. J. Renkema, W. R. Rossen, Optimal design criteria for SAG foam processes in heterogeneous reservoirs, in: 2009 SPE EUROPEC/EAGE Annual Conference and Exhibition, June 8-11, 2009, Amsterdam, The Netherlands, Society of Petroleum Engineers, 2009.

[8] K. Ma, R. Liontas, C. A. Conn, G. J. Hirasaki, S. L. Biswal, Visualization of improved sweep with foam in heterogeneous porous media using microfluidics, Soft Matter 8 (41) (2012) 10669-10675.

[9] A. R. Kovscek, H. J. Bertin, Estimation of foam mobility in heterogeneous porous media, in: SPE/DOE Improved Oil Recovery Symposium, April 13-17, 2002, Tulsa, Oklahoma, Society of Petroleum Engineers, 2002.

[10] J. Tsau, H. Yaghoobi, R. B. Grigg, Smart foam to improve oil recovery in heterogeneous porous media, in: 1998 SPE/DOE Improved Oil Recovery Symposium, April 19-22, 1998, Tulsa, Oklahoma, Society of Petroleum Engineers, 1998.

[11] P. Grassia, E. Mas-Hernández, N. Shokri, S. J. Cox, G. Mishuris, W. R. Rossen, Analysis of a model for foam improved oil recovery, J. Fluid Mech. 751 (2014) 346-405.

[12] D. Shan, W. R. Rossen, Optimal injection strategies for foam IOR, SPE Journal 9 (2) (2004) 132-150.

[13] E. Mas-Hernández, P. Grassia, N. Shokri, Foam-improved oil recovery: Modelling the effect of an increase in injection pressure., Eur. Phys. J. E 38 (2015) 67. 
[14] E. Mas-Hernández, P. Grassia, N. Shokri, Foam improved oil recovery: Foam front displacement in the presence of slumping., Colloids and Surfaces A: Physicochemical and Engineering Aspects 473 (2015) 123-132.

[15] R. M. de Velde Harsenhorst, A. S. Dharma, A. Andrianov, W. R. Rossen, Extension and verification of a simple model for vertical sweep in foam surfactantalternating-gas displacements, SPE Reservoir Evaluation \& Engineering 17 (3) (2014) 373-383.

[16] C. Torres-Ulloa, Predicción del frente espuma-petróleo en coordenadas Eulerianas, Master's thesis, Universidad Católica de Temuco, in Spanish (2015).

[17] P. Grassia, C. Torres-Ulloa, S. Berres, E. Mas-Hernández, N. Shokri, Foam front propagation in anisotropic oil reservoirs, Eur. Phys. J. E 39 (2016) 42. 


\begin{tabular}{cccc}
\hline$t_{D}$ & $k_{s}=0.3$ & $k_{s}=0.4$ & $k_{s}=0.5$ \\
& $n_{s}=3$ & $n_{s}=3$ & $n_{s}=3$ \\
\hline 0.1 & 0.0299 & 0.0391 & 0.0504 \\
0.2 & 0.0403 & 0.0535 & 0.0700 \\
0.3 & 0.0467 & 0.0671 & 0.0866 \\
\hline \hline$t_{D}$ & $k_{s}=0.3$ & $k_{s}=0.3$ & $k_{s}=0.3$ \\
& $n_{s}=3$ & $n_{s}=4$ & $n_{s}=5$ \\
\hline 0.1 & 0.0298 & 0.0285 & 0.0277 \\
0.2 & 0.0400 & 0.0392 & 0.0395 \\
0.3 & 0.0472 & 0.0474 & 0.0479 \\
\hline
\end{tabular}

Table 1: Root mean square displacement between homogeneous and heterogeneous fronts varying parameter $k_{s}$ and/or $n_{s}$. 


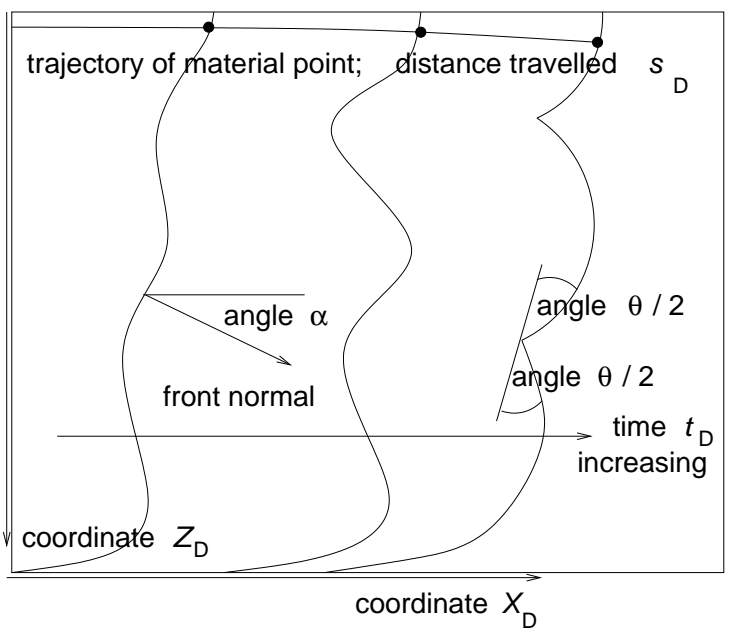

Figure 1: Schematic for the foam front displacement in a two-dimensional heterogeneous reservoir (in terms of $X_{D}$ vs $Z_{D}$ coordinates) as function of time. Snapshots of the foam front at different times $t_{D}$ are sketched. Here $s_{D}$ is the distance travelled by a material point on the front, and $\alpha$ is the angle between the normal to the front and the horizontal. Permeability differences affect the shape of the foam front (causing it to develop concavities and convexities), and are described using equation (3) or (4). A sharp concave corner is considered to have formed when the angle $\theta$ through which the front tangent turns at the concavity exceeds a certain critical value. 
(a)
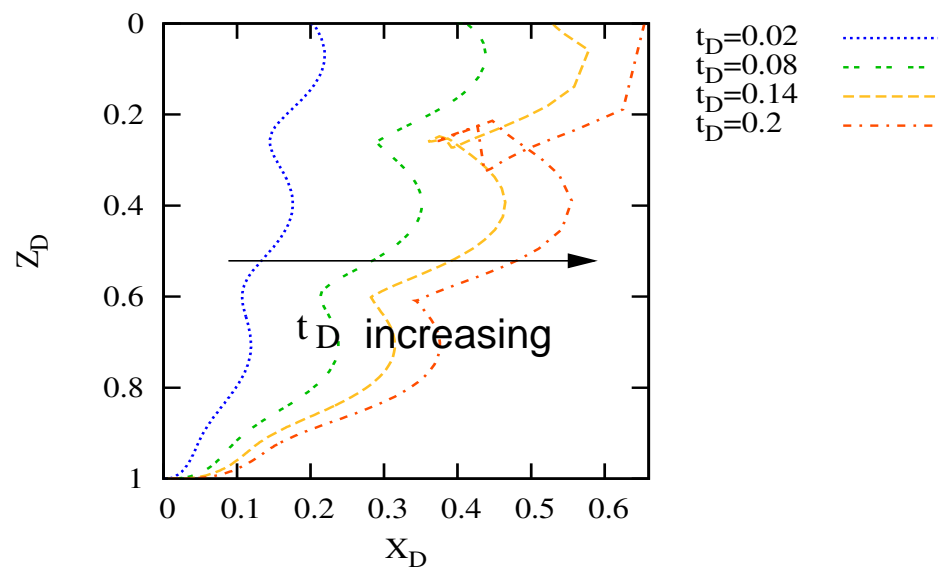

(b)
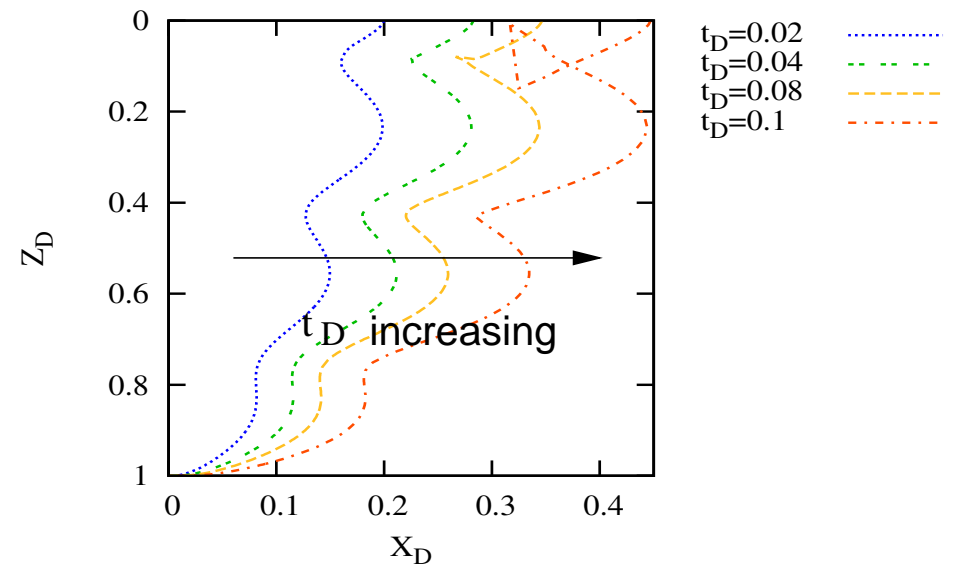

Figure 2: Foam front shape for various times $t_{D}$ (with $t_{D}$ increasing from curve to curve from left to right) for a stratified reservoir with $k_{s}=0.3$ and $n_{s}=3$ using (a) equation (3) for $J\left(Z_{D}\right)$ or (b) equation (4) for $J\left(Z_{D}\right)$. For these data, no correction is applied to velocities in concave regions of the front. 
(a)

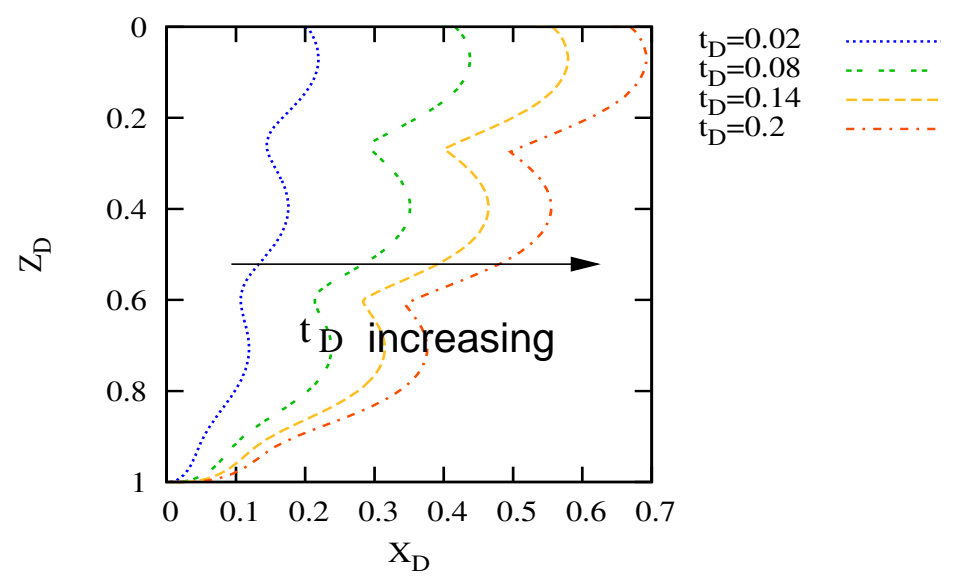

(b)

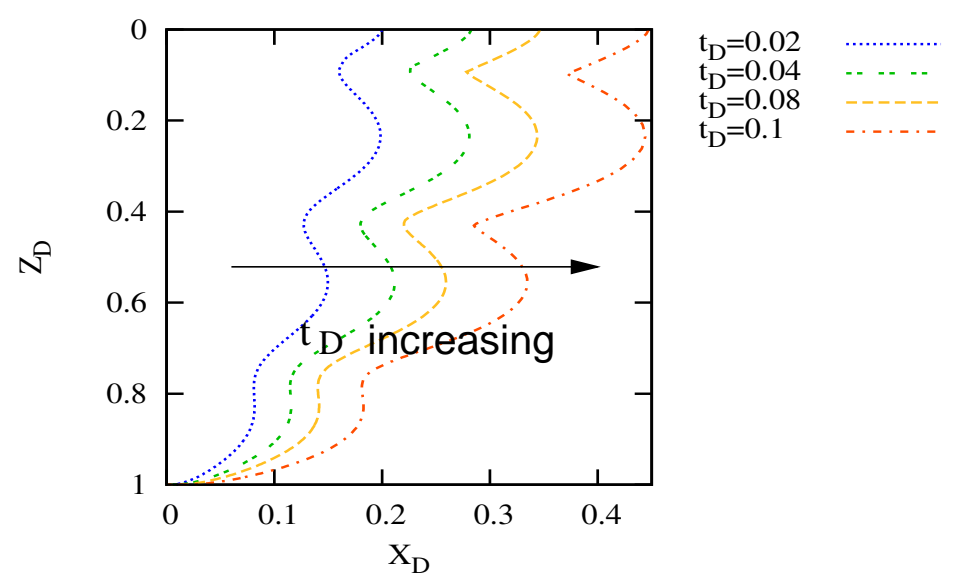

Figure 3: Foam front shape for a stratified reservoir for various times $t_{D}$ (with $t_{D}$ increasing from curve to curve from left to right), correcting velocities on concavities, regridding the front, and using (a) equation (3) for $J\left(Z_{D}\right)$ or (b) equation (4) for $J\left(Z_{D}\right)$. 


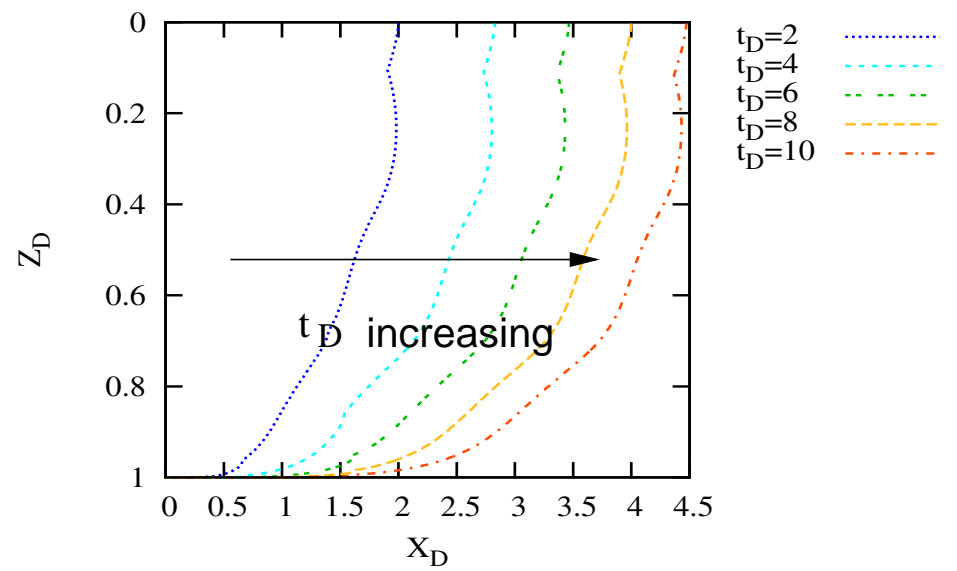

Figure 4: Foam front shape for longer time and using equation (4) for $J\left(Z_{D}\right)$. 
(a)

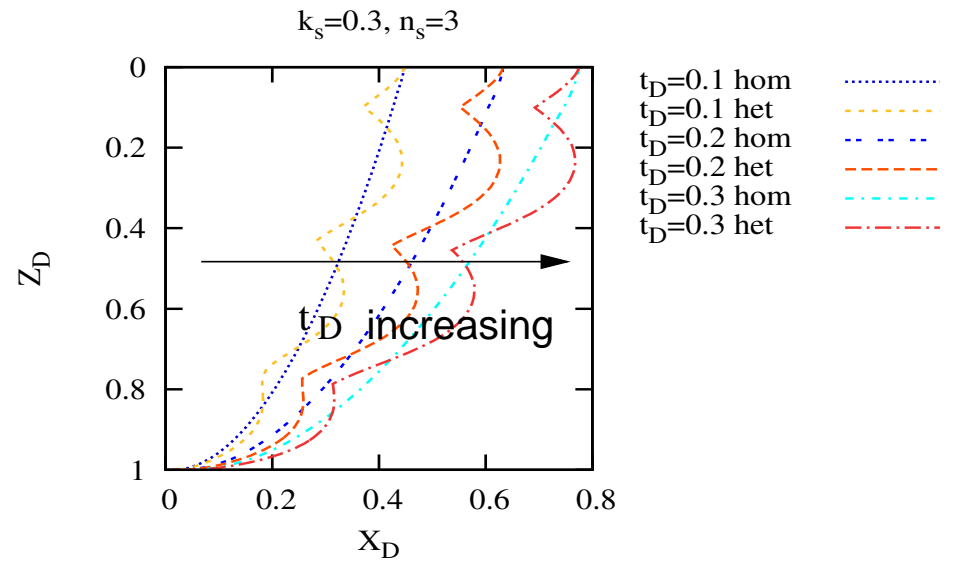

(b)

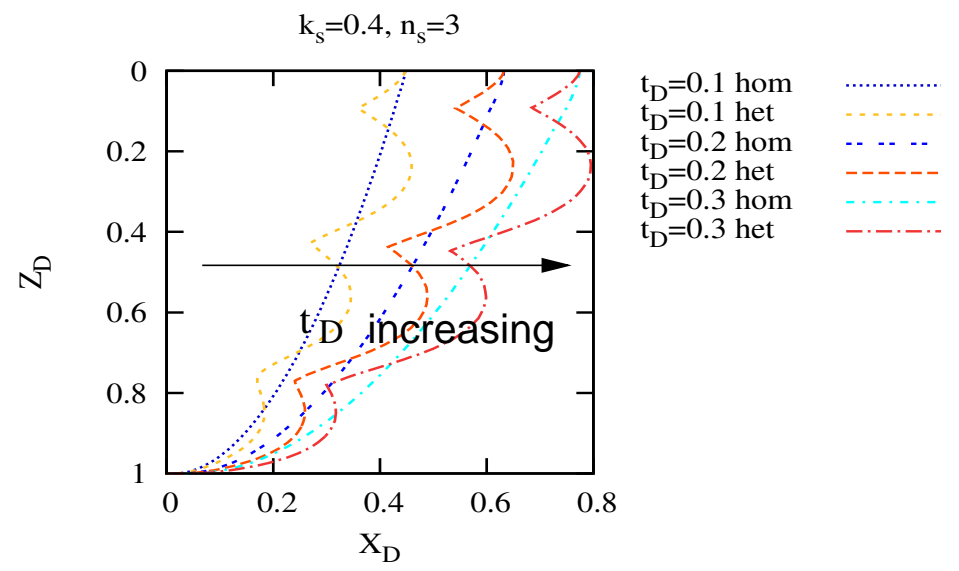

(c)

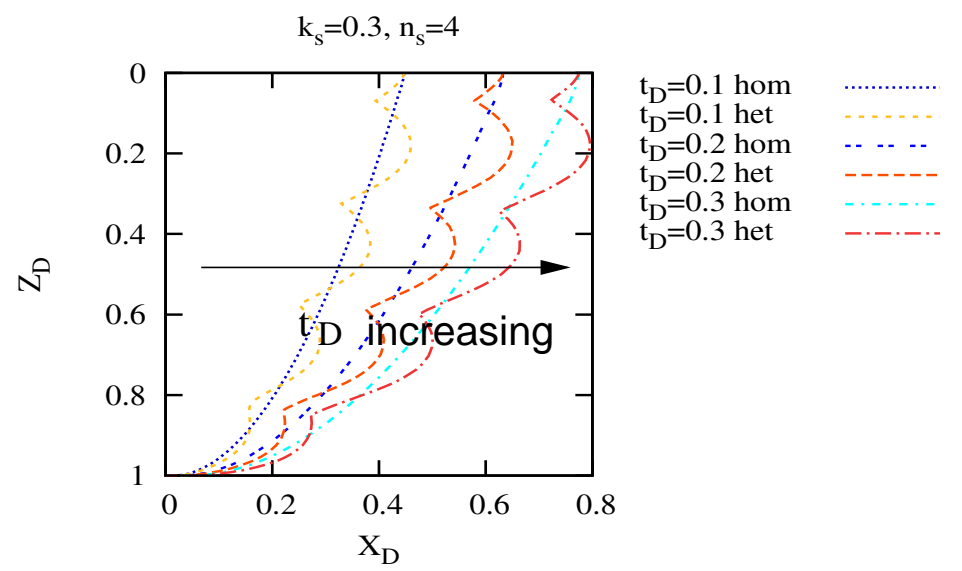

Figure 5: Displacements for homogeneous and heterogeneous fronts (a) $k_{s}=0.3, n_{s}=3$, (b) $k_{s}=0.4, n_{s}=3$, and (c) $k_{s}=0.3, n_{s}=4$. 
(a)

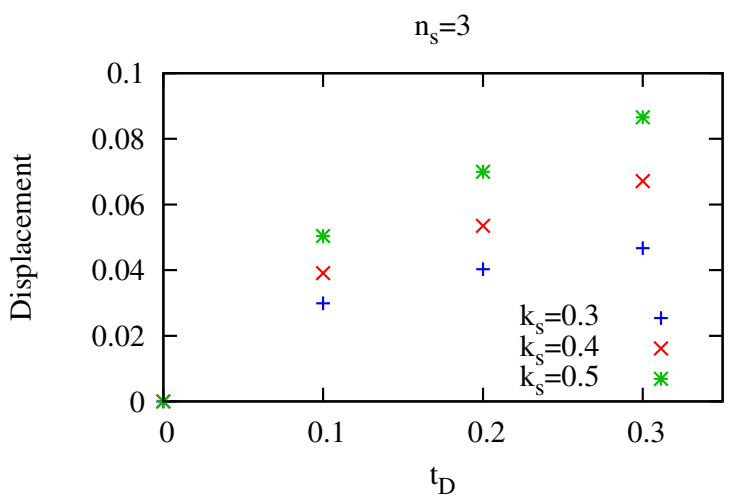

(b)

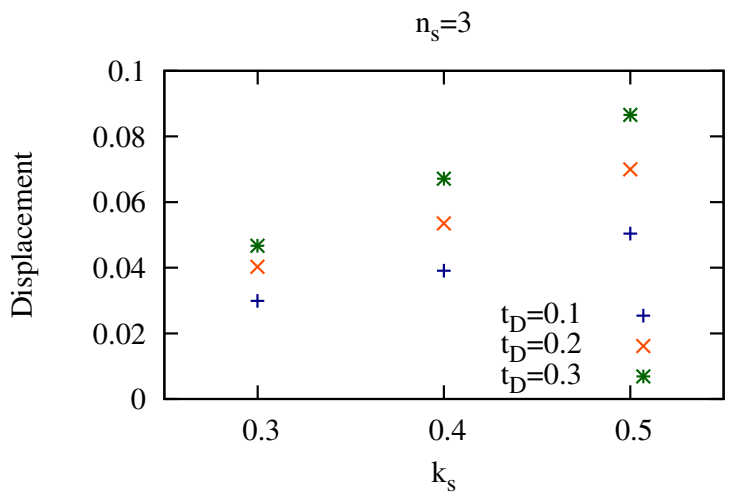

Figure 6: Root mean square displacement (a) vs time for various $k_{s}\left(k_{s}=0.3,0.4,0.5\right.$, with $n_{s}=3$ ), and (b) vs $k_{s}$ for various time $\left(t_{D}=0.1,0.2\right.$, and 0.3 still with $\left.n_{s}=3\right)$. 
(a)

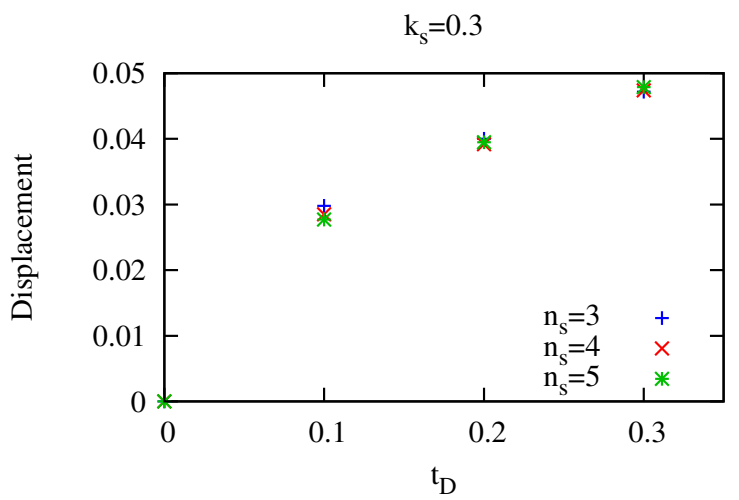

(b)

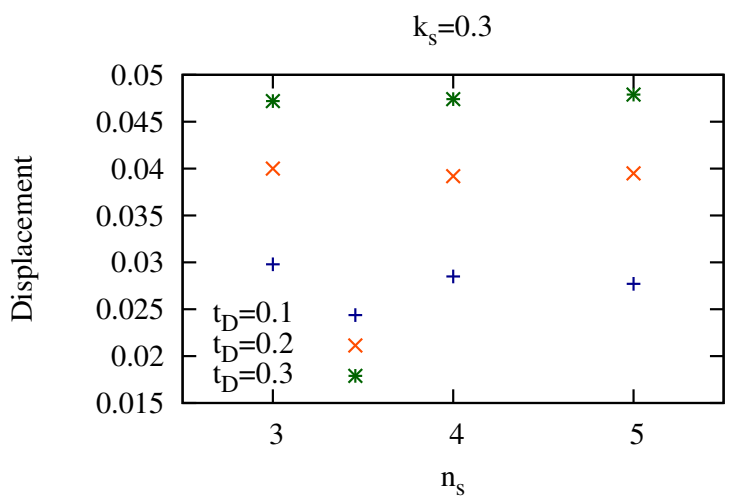

Figure 7: Root mean square displacement (a) vs time, for various $n_{s}$ (with $k_{s}=0.3$ ), (b) vs $n_{s}$ at various times $t_{D}=0.1, t_{D}=0.2$, and $t_{D}=0.3$. 


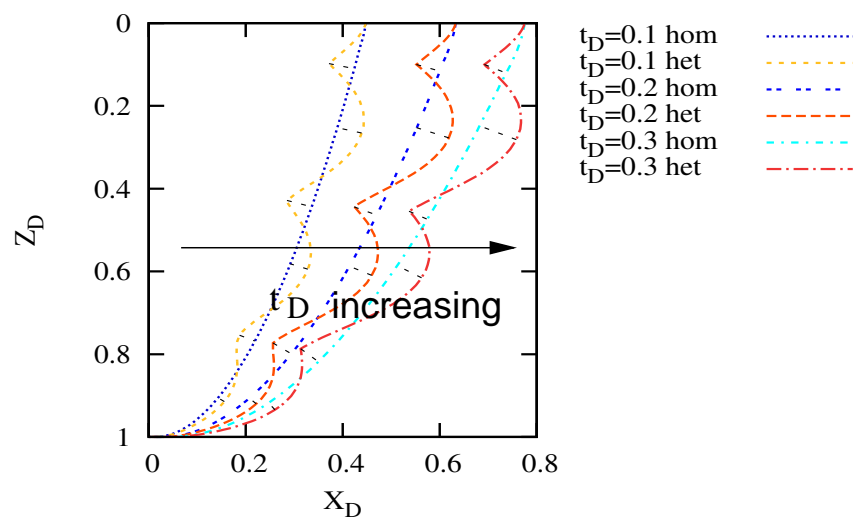

Figure 8: Homogeneous and heterogeneous foam fronts at $t_{D}=0.1,0.2$ and 0.3 (with $k_{s}=0.3$, $n_{s}=3$ ). Segments which are normal to the homogeneous curves and join heterogeneous points for local maxima and minima are also shown. 
(a)

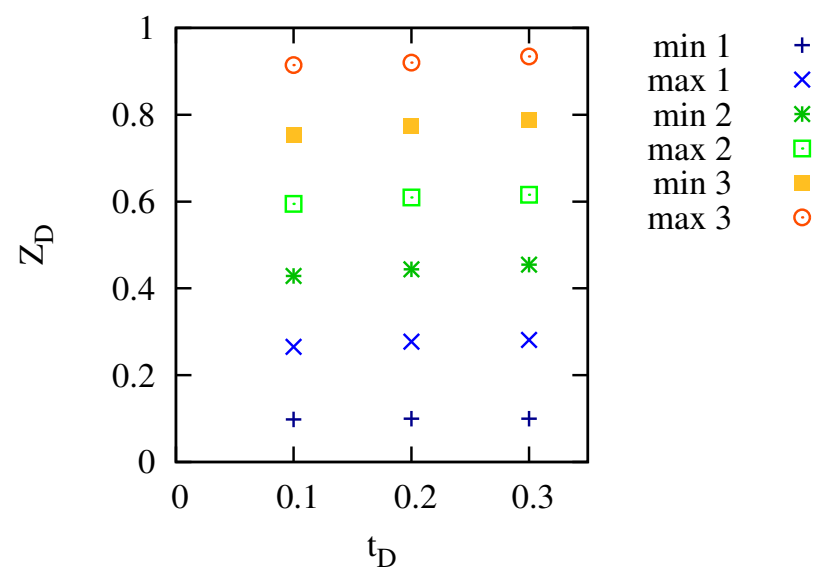

(b)

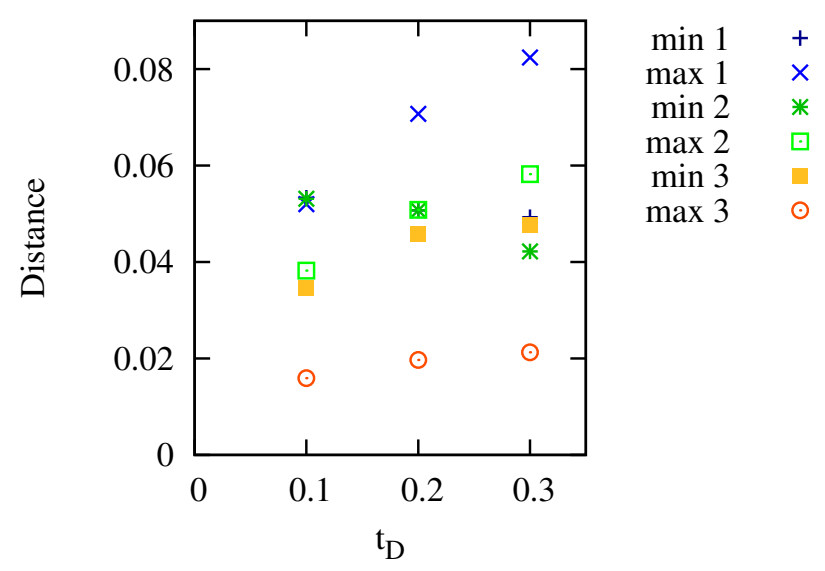

Figure 9: (a) Vertical point position for local minima and maxima from the top (min 1) to the bottom $(\max 3)$ of the front at times $t_{D}=0.1,0.2$ and 0.3 . (b) Distance between local minimum and maximum points and the homogeneous front. 


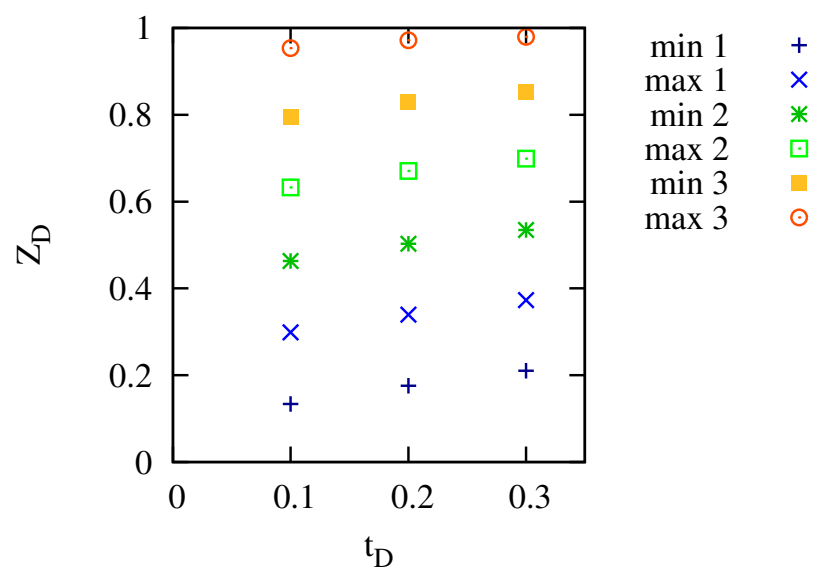

Figure 10: Vertical point position at times $t_{D}=0.1,0.2$ and 0.3 for selected material points in a homogeneous system which at early times start off near local minima and maxima in a corresponding heterogeneous system, numbered from the top (min 1$)$ to the bottom (max 3 ). 


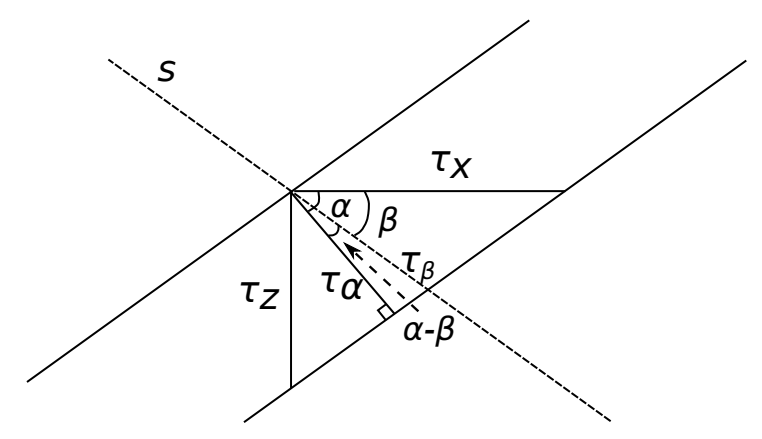

Figure 11: Zoomed view of the front showing schematically the angles $\alpha$ and $\beta$ for foam displacement within an anisotropic reservoir taken from [15]. Here $\alpha$ is the angle for the normal to the front and $\beta$ gives the direction of the front movement along the path that the material point follows. The front thickness measured along the direction $\beta$, which we denote $\tau_{\beta}$ grows proportional to the path length $s$ (dimensional variable analogous to $s_{D}$ ). Different values for front thickness $\tau$ are however determined in the directions $x, z$ and $\alpha$. 
(a)

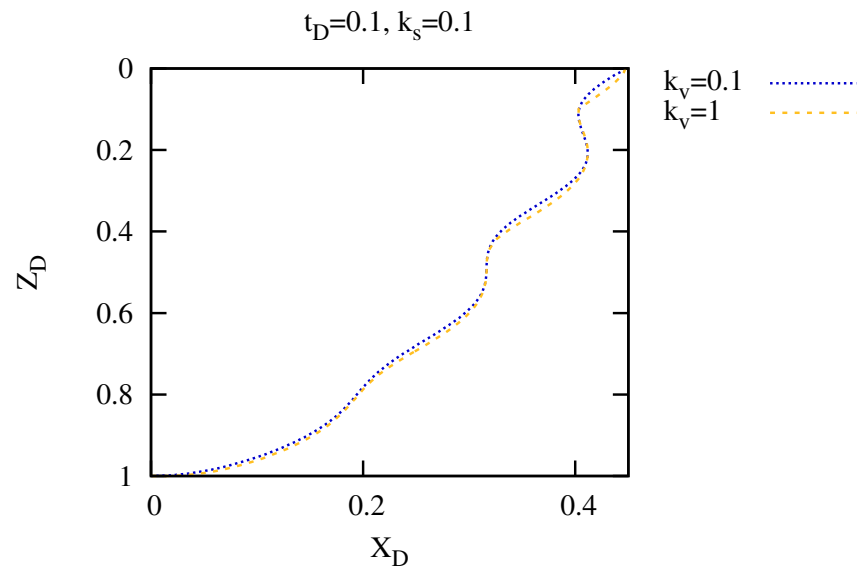

(b)

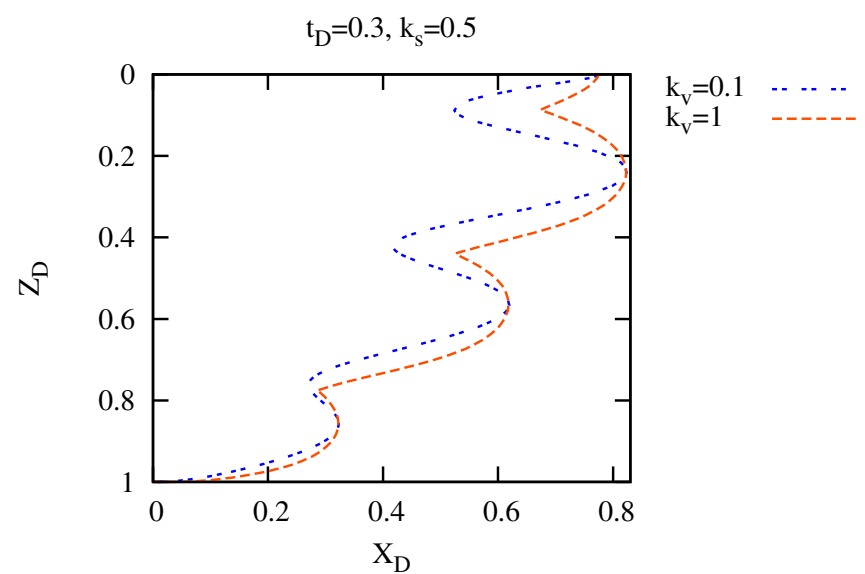

Figure 12: Fronts for heterogeneous anisotropic displacement varying $k_{v}$ (a) $t_{D}=0.1, k_{s}=0.1$ (b) $t_{D}=0.3, k_{s}=0.5$. 
(a)

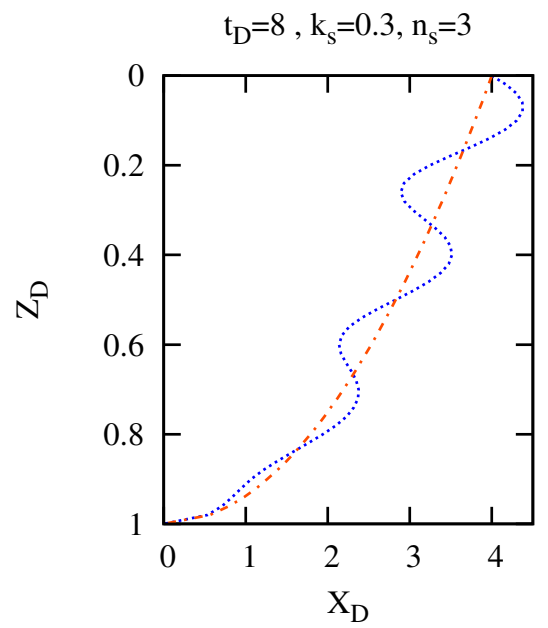

Heterogeneous $\quad$.................. Homogeneous

(b)

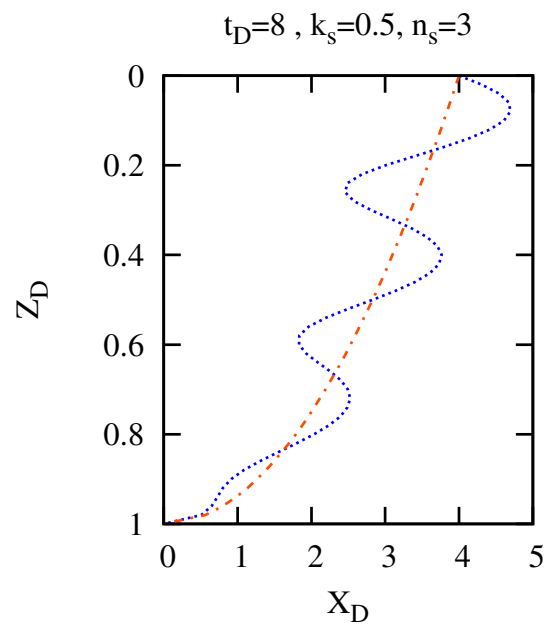

Heterogeneous

Homogeneous

(c)

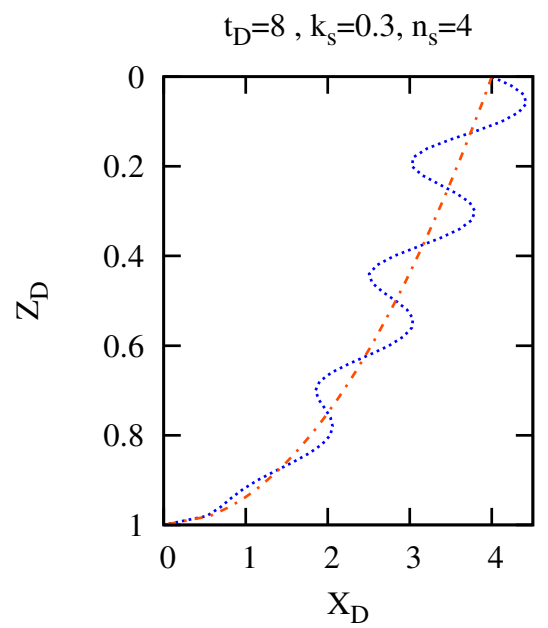

Heterogeneous

Homogeneous

Figure 13: Analytical results at $t_{D}=8$ for homogeneous and heterogeneous foam fronts when vertical permeability is zero. (a) $k_{s}=0.3, n_{s}=3$, (b) $k_{s}=0.5, n_{s}=3$, (c) $k_{s}=0.3, n_{s}=4$. 


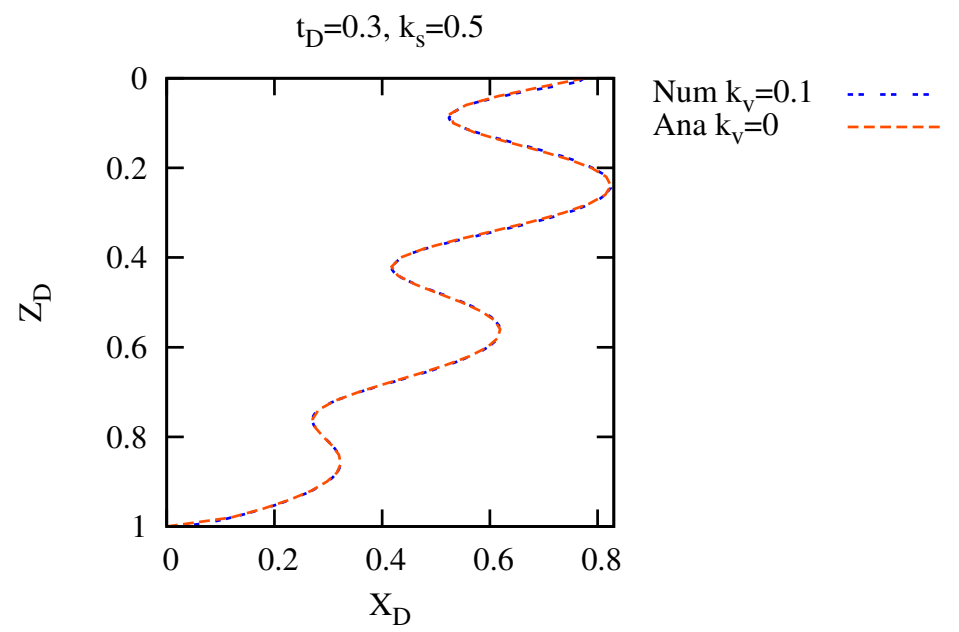

Figure 14: Heterogeneous anisotropic front displacement for numerical results with $k_{v}=0.1$ and analytical results when vertical permeability is zero. 